Relations industrielles

Industrial Relations

\title{
The Dynamics of Management, Waino W. Suojanen, Holt, Rinehart and Winston Inc., Toronto, 1966. 186 pages.
}

\section{Jean Sexton}

Volume 21, numéro 3, 1966

URI : https://id.erudit.org/iderudit/027708ar

DOI : https://doi.org/10.7202/027708ar

Aller au sommaire du numéro

Éditeur(s)

Département des relations industrielles de l'Université Laval

ISSN

0034-379X (imprimé)

1703-8138 (numérique)

Découvrir la revue

Citer ce compte rendu

Sexton, J. (1966). Compte rendu de [The Dynamics of Management, Waino W. Suojanen, Holt, Rinehart and Winston Inc., Toronto, 1966. 186 pages.] Relations industrielles / Industrial Relations, 21(3), 461-462.

https://doi.org/10.7202/027708ar

Tous droits réservés (C) Département des relations industrielles de l'Université Laval, 1966
Ce document est protégé par la loi sur le droit d'auteur. L’utilisation des services d'Érudit (y compris la reproduction) est assujettie à sa politique d'utilisation que vous pouvez consulter en ligne.

https://apropos.erudit.org/fr/usagers/politique-dutilisation/ 
voulons surtout souligner ici le rajeunissement qu'o subi la revue au début de l'année 1966. En adoptant un format du type Times, U.S. News and World Report, ou Express, on a pu introduire une plus vaste aération des textes, mettre en exergue les titres d'articles, modifier le caractère des séries statistiques, ce qui en rend la lecture beaucoup plus facile. Nous tenons d̀ féliciter l'équipe de rédaction pour cette présentation plus vivante.

Québec/Travail, Ministère du Travail, Québec, P.Q.

Le Bulletin mensuel d'information publié par le Service d'information du Ministère du Travail a fait place au Journal du Travail. Comme plusieurs périodiques arborent des noms similaires, l'équipe de rédaction a décidé de transformer cette appellation. Le Journal, en janvier 1966, prend le nom de Québec/Travail. L'impression sur papier glacé permet une présentotion beaucoup plus soignée.

Québec/Travail se veut un reflet du dynamisme qui caractérise le monde du travail québecois. En plus de nous fournir une documentation statistique sur les principales activités du Ministère, sur la situation de l'emploi, le journal reproduit des textes de conférences importantes, des études sérieuses sur I'emploi, la répartition de la maind'oeuvre, le contenu des conventions collectives, etc.

Nous voulons aussi attirer l'attention sur Ic lutte qu'on y mène contre les anglicismes ou cutres déformations de mots anglais dans les relations de travail. Une terminologie française qui apparaît à tous les mois facilite I'utilisaton des termes français exacts. L'équipe pourrait peut-être ajouter une liste trimestrielle des publications récentes d'articles ou de volumes français couvrant le domaine des relations industrielles. Nous souhaitons beaucoup de succès et longue vie à cette publication surtout utile à ceux qui oeuvrent dans le domaine des relations industrielles.

\section{Lourent BELANGER}

Psycho-sociologie du travail, Pierre Jaccard, Etudes et Documents, Payot, Paris, 1966. 177 pages.

* Il faut s'attendre ò ce que le travail humain soit touché dans les formes, d'ailleurs en perpétuelle évolution, qu'il a prises depuis l'époque de l'artisanat, mais on ne voit pas $q u^{\prime} i l$ soit ou puisse être atteint dans sa nature profonde, dans les fonctions diverses qu'il remplit dans la vie individuelle et sociale. »

Pour exposer sa thèse, l'auteur présente une génèse de la notion du travail: il montre l'évolution de cette notion depuis la révolution industrielle en se servant d'exemples tirés de l'histoire et de situations contemporaines semblables à celles de cette époque. Jaccard insiste sur la nécessité du travail telle que ressentie par les travailleurs individuellement et collectivement. Au sujet des changements dans la nature du travail * ce qui va diminuer dans l'activité professionnelle ce n'est pas tellement le travail mais l'intensité de la contrainte du travail $₫$. Le travail apportera une plus grande part de liberté et de spontanéité au travailleur.

Dans une seconde partie, l'auteur explique que, avec des développements techniques de plus en plus rapides, la notion de travail demeurera tout aussi importante, mais deviendra aussi plus a intellectuelle $\$$. Le travailleur aura besoin de plus en plus d'éducation, à laquelle sont liés développement et progrès. Enfin Jaccard traite des implications de cette évolution sur le choix du métier par les étudiants, de l'orientation professionnelle et de la formation scolaire de ceux-ci. Notons que Jaccard est très bien placé pour traiter de cette notion, ayant déjà rédigé une « Histoire sociale du travail $\$$.

\section{Emile VALLEE}

\section{The Dynamics of Management, Waino W.} Suojanen, Holt, Rinehart and Winston Inc., Toronto, 1966. 186 pages.

Le caractère de la civilisation moderne ne fut grandement fixé que par l'influence jumelle de la démocratie et de la science. La théorie évolutioniste de l'administration considère la démocratie et la science comme étant des facteurs dynamiques intégrés à la fois dans la pratique et la théorie de l'administration. Selon le professeur Suojanen, la pratique administrative, la structure dé l'organisation et le style de leadership doivent être revisés pour rencontrer les exigences des institutions scientifiques. Pour ce faire, l'auteur développe une approche à la fois théorique et pratique de l'administration dans I'organisation scientifique. Cet ouvrage présente un thème très important à savoir l'intégration des valeurs de la démocratie et de la science dans la politique et les ospects administratifs de l'organisation scientifique. 
The Dynamics of Management, qui s'adresse à la fois au théoricien de l'administration et au * manager $\$$, comprend quatre parties inégales

Lo première partie (chap. 1-3) consiste en une présentation de matériaux de base et en une critique de la théorie fonctionnelle de l'administration en tant qu'appliquée à l'organisation scientifique. L'auteur poursuit (chap. 4-9) en tentant d'intégrer un certain nombre de théories de l'administration en un seul concept interdisciplinaire. Puis on retrouve une discussion (chap. 10-12) ou sujet de l'application des concepts évolutionistes de l'administration à l'entreprise décentralisée. Finalement (chap. 13-17) le professeur Suojanen prétend que le système libéral et la théorie évolutioniste de l'administration ont beoucoup en commun et tendront à converger dans le futur.

Le présent ouvrage révèle donc un aspect nouveau de l'administration et réflète la grande importance de la recherche américaine dans ce domaine.

\section{Jean SEXTON}

Soviet Management With Significant American Comparisons, Barry M. Richmon, Prentice-Hall, Engelwood Cliffs, New Jersey, 1965, 279 pages.

Dès le début de son ouvrage, l'auteur note qu'il existe deux façons de traiter de l'économie soviétique. La première serait une vue générale de l'économie de I'U.R.S.S. Le second type d'ouvrage serait une vue microéconomique en considérant les problèmes des institutions et des gens à des niveaux plus bos. Ces travaux traitent très peu de l'administration soviétique, mais il n'existe que très peu d'analyse réelle.

Le présent ouvrage a été présenté dans le but de faire le pont entre ces deux types d'études. Ceci a nécessité un examen complet des milieux politique, économique et culturel qui influencent l'administration soviétique. Des interviews personnels et une recherche intense à travers I'U.R.S.S. ont fourni les informations nécessaires pour étoblir une comparaison significative entre les administrations russe et américaine.

Ecrivant à la fois pour le spécialiste et le non-spécialiste, le Dr Richman s'intéresse d'abord à l'administration de l'entreprise industrielle soviétique, puis à la marche de l'économie de I'U.R.S.S. considérée comme un tout, mais seulement en tant qu'influence sur l'administration de l'entreprise.
La première partie de cet ouvrage présente une brève comparaison entre les U.S. et I'U.R.S.S. Puis I'auteur considère les fonctions de l'industrie soviétique en examinant de plus près l'entreprise industrielle et son administration. Le troisième chapitre traite surtout des problèmes administratifs de l'industrie russe. Finalement le $\mathrm{Dr}$ Richman analyse la réforme administrative soviétique.

Cet ouvrage constitue donc une étude originale de l'administration soviétique moderne qui nous est présentée en comparaison avec les U.S.

\section{Jean SEXTON}

The Management of Human Relations, Saul W. Gellerman, Holt, Rinehart and Winston of Canada, Toronto, 1966. 138 pages.

$\mathrm{Ce}$ volume fait partie d'une collection introduite dernièrement par la maison Holt, Rinehart and Winston. L'auteur de chaque volume essaie de réaliser une synthèse des travaux parus à date sur un point important en administration de l'entreprise, direction du personnel et relations industrielles. Management Science and Management Controls ouvre la série. Organization Theory, The Management of Human Relations and Executive Controls traitent de l'utilisation efficace des ressources humaines. Industrial Relations couvre les politiques directoriales dans le domaine des relations syridicat-direction. Integrated Manufacturing présente une vue générale de l'administration de la production. Enfin, International Management couvre une région frontière en administration, celle de la direction des entreprises dans les pays autres que le Canada et les Etats-Unis.

Gellerman, I'auteur du volume qui fait l'objet de cette recension, est un proticien de la direction du personnel à l'emploi de la compagnie IBM. Son exposé sur l'origine et le contenu des relations humaines dans l'entreprise tente d'allier la théorie à la pratique. En traitant, dans son introduction, de l'importance des conséquences de "bonnes" relations humaines et les difficultés que comporte la mise en opplication des résultats de recherche, Gellerman cherche à dissiper certains mythes concernant le moniement des hommes. Brandir l'arme des récompenses monétaires et des punitions ou donner de temps à autre une tope amicale et appeler quelqu'un par son prénom sont deux méthodes inadéquates pour inciter les gens à fournir la contribution qu'on atteno d'eux. "Coercition and compensation are still with us, of course, and they probably 